\title{
La expansión internacional de la industria vitivinícola chilena. Estudio empírico de los factores y nivel exportador
}

\section{International expansion of the chilean wine industry: ann empirical study concerning the factors and exportation level}

\author{
SAMMY LiBERMAN ${ }^{1}$ \\ VERÓNICA BAENA ${ }^{2}$ \\ FÁtima Moreno ${ }^{3}$
}

Universidad Andrés Bello / Universidad Europea de Madrid

Recibido el 9 de septiembre de 2009, aceptado el 13 de diciembre de 2009

$\mathrm{N}^{\mathrm{o}}$ de clasificación JEL: F0 y F2

DOI: $10.5295 / \mathrm{cdg} .090174 \mathrm{sl}$

Resumen:

Este trabajo propone una serie de factores como determinantes del nivel de expansión internacional de la industria vitivinícola chilena: i) tamaño de la empresa; ii) gestión de la calidad; iii) tecnologías de la información y la comunicación; iv) planificación de la gestión internacional, y finalmente, v) adaptación de la estrategia de marketing internacional. El contraste empírico sobre la base de datos de exportación de la industria vitivinícola chilena entre los años 2004 y 2007, permitió confirmar seis de las siete hipótesis planteadas. Este estudio concluye con un apartado destinado a resumir las principales contribuciones teóricas y prácticas, así como las limitaciones y futuras líneas de investigación.

Palabras clave:

Expansión internacional, Industria Vitivinícola Chilena.

Abstract:

This study proposes a set of variables which are decisive in the international expansion of the Chilean Wine Industry: i) company size; ii) quality management; iii) communication and information technologies; iv) international management planning; and finally, v) implementation of the international marketing strategy. Results based on data between 2004 and 2007 allow to confirm six of the seven formulated hypotheses. The implications of these findings for theory and practice are discussed, as well as the main limitations. Finally, we offer some recommendations for further research.

Keywords:

International expansion, Chilean Wine Industry.

${ }^{1}$ Universidad Andrés Bello. Facultad de Economía y Empresas. C/Pastor Fernández Concha 700, Las Condes, (Santiago de Chile-Chile). E-mail: s.liberman@uandresbello.edu

${ }^{2}$ Universidad Europea de Madrid. Departamento de Empresa. C/Tajo, s/n. Urb. El Bosque 28670-Villaviciosa de Odón (Madrid).E-mail: verónica.baena@uem.es

${ }^{3}$ Universidad Europea de Madrid. Departamento de Empresa. C/Tajo, s/n. Urb. El Bosque 28670-Villaviciosa de Odón (Madrid).E-mail: mfatima.moreno@uem.es 


\section{INTRODUCCIÓN}

El fenómeno de la internacionalización de empresas se ha esgrimido de muchas formas en la literatura, sin embargo ninguna de las aproximaciones teóricas y metodológicas parecen ser completamente definitivas para dar cuenta de la alta variedad y complejidad de interpretaciones y experiencias que ofrece dicho fenómeno. Así pues, la decisión de una empresa de expandirse internacionalmente pasa por el análisis y evaluación de numerosos aspectos y consideraciones. Por una parte, los beneficios potenciales tienen que ver con aspectos tales como la necesidad de ampliar la base de clientes para mejorar las economías de escala, la posibilidad de acompañar a clientes locales que demandan servicios internacionales en el extranjero, así como la eliminación de la dependencia hacia un único mercado (Jarillo y Martinez, 1991; Czinkota y Ronkainen, 2004). Por el contrario, los costes potenciales de expandirse internacionalmente se relacionan con aspectos tales como las restricciones legales o el desconocimiento de la cultura del país receptor de la inversión (Czinkota y Ronkainen, 2004; Cateora y Graham 2006).

Todas las consideraciones anteriores deben ser cotejadas en el momento de tomar una decisión de expansión internacional. Sin embargo y de acuerdo con Jarillo (1996), el verdadero punto de inflexión relacionado con el dilema de tener o no presencia fuera del país de origen estará relacionado con la correcta evaluación por parte de la empresa del valor añadido que puede generar dicha estrategia. En este sentido, aspectos como eficiencia, prestigio, aprendizaje y flexibilidad entre otros, debieran ser considerados como los verdaderos «detonantes» en la decisión de iniciar la estrategia de internacionalización.

Por otra parte, a la hora de expandirse hacia un nuevo mercado o conseguir un mayor grado de penetración en el que ya se opera, las empresas deben considerar un amplio conjunto de posibilidades de formas de expansión: i) adoptar estrategias de propiedad total (nueva implantación o adquisición); ii) joint ventures (mayoritarias o minoritarias); e incluso, iii) acuerdos contractuales (tales como contratos de gestión de servicios, licencia o exportación). Tal y como se recoge en la Tabla 1, cada uno de estas formas de entrada implica diferentes grados de propiedad y de control por parte de la matriz (Erramilli y Rao, 1993; Contractor y Kundu, 1998a; 1998b). La elección de la forma de entrada tendrá que ver en términos generales con los grados de riesgo, compromiso, control y retornos que la empresa desea involucrar en la expedición internacional (Contractor y Kundu, 1998a; 1998b; Pan y Tse, 2000; Brouthers y Brouthers, 2003; Czinkota y Ronkainen, 2004; entre otros).

\section{Tabla 1}

Formas de expansión empresarial

\begin{tabular}{|lll|}
\hline Alto Control & \multicolumn{1}{c|}{ Propiedad } & $\longrightarrow$ No Propiedad \\
${ } }$ & Joint Venture & Contrato de Gestión de Servicios/Leasing \\
\hline
\end{tabular}

Fuente: Elaboración propia a partir de Contractor y Kundu (1998b) y Pan y Tse (2000). 
Con relación a lo anteriormente expuesto, este trabajo pretende contribuir a la literatura a través de la elaboración de un modelo conceptual que ayude tanto a los académicos como a los empresarios a comprender y predecir el grado de internacionalización de las empresas vinculadas a la industria vitivinícola chilena, un sector de altísima relevancia para dicho país y de creciente presencia internacional. De hecho, atendiendo a los datos publicados en 2008 por la Organización Internacional de la Viña y el Vino (OIV), Chile pasó de exportar MM 0,8 de hectolitros de vino el año 1995 a MM 4,2 de hectolitros el año 2005.

Además, la industria vitivinícola chilena es una de las de mayor dinamismo y proyección internacional, representando para dicho país la quinta fuente de generación de divisas a la fecha de realización del estudio. Junto a ello, Chile es considerado uno de los 10 primeros países productores de vino del mundo y uno de los tres productores con mayor crecimiento en los últimos 5 años en lo que a volumen exportador se refiere ${ }^{4}$. De este modo, Chile ocupa la $5^{\text {a }}$ posición como país exportador a nivel mundial — sólo por detrás de Italia, España, Francia y Australia - , así como la $2^{\circ}$ posición en cuanto a la relación exportación/ producción ${ }^{5}$. Por último, es preciso señalar que Chile es el único país exportador perteneciente al grupo de los 5 principales países exportadores de vino que además de aumentar sus exportaciones de vino, aumentó paralelamente su nivel de producción vitivinícola así como el volumen de superficie plantada de vid para la elaboración de vino.

Para alcanzar los objetivos antes expuestos, en el siguiente apartado realizaremos una revisión de literatura sobre las principales aportaciones académicas realizadas sobre el proceso de internacionalización empresarial para proponer a continuación, una serie de hipótesis. Seguidamente, describiremos la metodología seguida en nuestra investigación empírica, la muestra de empresas utilizadas, así como las variables definidas. En la sección posterior se procederá a mostrar y explicar los resultados obtenidos tras el contraste estadístico de las hipótesis formuladas en el trabajo. Finalmente, concluiremos con un apartado destinado a resumir las ideas principales del trabajo, sus contribuciones (académicas y prácticas), así como las limitaciones y futuras líneas de investigación.

\section{REVISIÓN DE LITERATURA Y FORMULACIÓN DE HIPÓTESIS}

El tamaño de la empresa y su relación con la expansión internacional ha sido ampliamente analizado en la literatura. Si bien existen conclusiones de algunos autores (Reid,

\footnotetext{
${ }^{4}$ Este trabajo considera el volumen exportador como proxy del grado de internacionalización de la industria vitivinícola chilena por los siguientes motivos: En primer lugar, en primer lugar, debido a la complejidad que supondría pretender abordar (sin información suficiente) la totalidad de los posibles modos de entrada a través de los que la empresa vitivinícola chilena puede entrar en nuevos mercados - exportación, cooperación, alianzas e inversión productiva en el exterior, entre otros - (Root, 1994; Durán, 1994). En segundo lugar, porque la actividad comercial agropecuaria chilena en el exterior (categoría en la cual se enmarca la actividad vitivinícola) está representada en su inmensa mayoría por la actividad exportadora y no por las inversiones en el exterior. Finalmente, cabe señalar que los volúmenes involucrados anualmente son en promedio 15 veces superior en el caso de las exportaciones que en el caso de las inversiones en el exterior (MMUS\$ 3.134 y MMU\$ 199 respectivamente, según datos publicados por Estadísticas e Indicadores de la Cámara de Comercio de Santiago de Chile A.G. -2008).

${ }^{5}$ Si bien el promedio mundial de la relación (exportaciones/producción) es del 33\%, tanto Chile como Australia registran los dos mayores porcentajes en dicha relación, alcanzando un $74 \%$ y $81 \%$, respectivamente. Fuente: OIV 2008.
} 
1982, Labeaga y Martínez, 1994; Alonso y Donoso, 2000, entre otros) que han corroborado una relación inversa entre el tamaño empresarial y la propensión exportadora, así como también una relación poco significativa entre dichas variables (Czinkota y Ronkainen, 2004); el mayor volumen de evidencias investigadoras favorece la relación positiva entre dichas variables.

En este sentido, varios trabajos señalan que dado que las empresas de mayor tamaño son capaces de orientar de mejor manera su acción comercial, lo que les permite actuar como verdaderos polos de atracción de recursos humanos y reducir la rotación de personal (Alonso y Donoso, 1998; Alfredo, 2001; Bohlander, Snell y Sherman, 2001, entre otros). Ello facilitaría la interacción productiva entre el personal y su empresa (Cateora y Graham, 2006), lo cual redundaría en un mayor nivel de desarrollo para la gestión internacional (véase Bonaccorsi, 1992; Calof, 1993; 1994; Calof y Viviers, 1995; Dean, Menguc y Myers, 2000; Melle y Raymond, 2001; entre otros).

Por otra parte, investigaciones realizadas a empresas exportadoras manufactureras en Sudamérica por Moori-Koenig y otros $(2005 ; 2007)$ también evidenciaron una relación positiva entre tamaño y grado de internacionalización de las empresas argentinas y colombianas. Desde esta perspectiva y a la luz de los resultados de los estudios anteriores, presentamos la siguiente hipótesis:

\section{H1: Las empresas con mayor tamaño, presentan un mayor grado de internacionali- zación}

No es poca la literatura que señala que el proceso empresarial, se potencia y robustece cuando es respaldado por algún estándar internacional que asegure la calidad (véase por ejemplo, Merino y Moreno, 1996; Merino, 1998; Anderson, Daly y Johnson, 1999). Lo anterior nada dice de la elección y selección del proceso productivo ideal, sin embargo la obtención de un certificado internacional de calidad actúa como una garantía en la resolución de la mayoría de los problemas que afrontan oferentes y demandantes de productos/ servicios. Este es un aspecto importante para oferentes de países en desarrollo o cuyo producto no es muy conocido y que buscan insertar su oferta exportadora en mercados con mayor nivel de exigencia (Castagnino, 2006; Cerviño, 2006) 6

A pesar de lo anteriormente expuesto, algunos autores señalan la existencia de costes hundidos en las decisiones de exportación, entre los cuales destacan los costes de transporte y aquellos asociados a la logística de venta, así como los costes vinculados a la obtención de estándares internacionales de producción (Roberts y Tybout, 1997; Bernard y Jensen, 2004; Maurel, 2009). Dicha afirmación se deriva del hecho de que sólo las empresas que gozan de una productividad por encima del promedio, estarían en condiciones de afrontar los costes adicionales que implica insertarse en el mercado internacional. Siguiendo la argumentación anterior, se puede afirmar que la presencia de certificados de estándares de

\footnotetext{
${ }^{6}$ La presencia de certificados de calidad (por ejemplo, ISO 9001) en ocasiones resulta una forma de resolver el problema de la asimetría de información que regularmente afrontan oferentes y demandantes. Este coste adicional realizado para «señalizar» adecuadamente los bienes producidos por la empresa, puede ser mejor absorbido a priori por aquellas compañías que detentan un mayor grado de productividad al lograr «prorratear» de mejor manera dicho coste entre las unidades producidas.
} 
calidad favorece el mayor grado de expansión internacional. Por lo tanto, presentamos la siguiente hipótesis:

\section{H2 Las empresas que enfatizan en la utilización de estándares internacionales de gestión de la calidad, presentan un mayor grado de internacionalización}

A lo largo de los últimos años, el denominado set de nuevas tecnologías de la información y de la comunicación (TIC) se ha ido incorporando de forma paulatina al entorno empresarial. Además, atendiendo a los resultados obtenidos en trabajos anteriores (DíazChao, 2003; Dehning y Strapoulos, 2003; Alderete, 2007, entre otros) ha quedado demostrado que las TIC pueden incrementar la competitividad de cualquier negocio. Por otra parte, Crick y Jones (2000) sugieren que la comprensión del espacio y las distancias favorecen más a las pequeñas empresas -inclusive en mercados complejos- dado que las grandes empresas tienen recursos suficientes para incrementar su visibilidad y sus contactos a través de otros mecanismos como por ejemplo, las ferias internacionales.

Así pues, a través del uso de tecnologías de la comunicación, las empresas están en condiciones de presentar sus productos en mercados distantes y mejorar la estrategia de marketing internacional (Kirpalani y Macintosh, 1980). No obstante, Yoguel y otros (2004) sugieren que la disposición de estas herramientas (TIC) no es suficiente si no le acompaña un cambio a nivel técnico y organizacional que permita la generación y circulación del conocimiento del tipo smart $\operatorname{mobs}^{7}$, del cual la innovación es la versión empresarial. En otras palabras, el proceso de internacionalización está en parte determinado por la posesión de recursos tecnológicos y de la comunicación que permiten a las empresas extenderse más allá de sus fronteras (Panayi y Trigeorgis, 1998; Bernal y Pajares, 2006; Loane, 2006; Nieto, 2006; García-Canal, Rialp-Criado y Rialp-Criado (2007). Desde esta perspectiva, la incorporación a la empresa de las tecnologías de la información (TIC) como mecanismos permanentes de gestión debería favorecer la presencia internacional y por ello, presentamos la siguiente hipótesis:

\section{H3: Las empresas que enfatizan en la utilización de tecnologías de la información y de la comunicación (TIC) presentan un mayor grado de internacionalización}

Los problemas más complicados al enfrentar ambientes culturales diversos del mercado internacional surgen del hecho de que no se puede aprender la cultura; hay que vivirla (Czinkota y Ronkainen, 2004). A este respecto, se habla de dos posturas opuestas sobre la manera de enfrentar la diversidad cultural. La primera sugiere que los negocios son actividades semejantes en todo el mundo, por lo que las empresas que operan en diferentes mercados no requieren de una adaptación especial (Harrison y Huntington, 2000; Stevens y Burns, 2005; Cateora y Graham, 2006). Sin embargo, la segunda corriente sostiene todo lo contrario al defender que la comprensión de las diferencias culturales es un aspecto crítico para competir con probabilidades de éxito en el terreno internacional (Stanton, Etzel y Walker, 2000).

\footnotetext{
${ }^{7}$ Smart MOBS (multitudes inteligentes). Concepto acuñado en Rheingold (2004).
} 
Así pues, desde la segunda perspectiva se afirma que las empresas que enfatizan en la formación y capacitación de sus recursos humanos están mejor preparadas para enfrentarse a la diversidad cultural inherente a cualquier negocio internacional (Black y Mandenhall, 1991, entre otros). En otras palabras, cuando se opera en mercados extranjeros es necesario adaptar y flexibilizar los métodos y formas de aprendizaje a las características del nuevo entorno (Neelankavil, Mathur y Zang, 2000). Además, las empresas que buscan un mayor compromiso internacional requieren de mayor flexibilidad en los procesos de entrenamiento del personal (Cateora y Graham, 2006). Por todo ello, siguiendo esta corriente formulamos la siguiente hipótesis:

\section{H4: Las empresas que enfatizan en la formación y capacitación de su personal pre- sentan un mayor grado de expansión internacional}

La morfología que adquiere la empresa exportadora también guarda relación con la forma en que ésta se organiza. Así pues, Alonso y Donoso (2000) concluyen que la presencia de procesos formales de planificación de las actividades de exportación afecta positivamente al desempeño exportador de las empresas. Dichos autores aluden a la idea de que la mayor formalidad en dichos procedimientos redunda en mayor grado de compromiso para con la actividad exportadora, así como mejor grado de organización.

Otros trabajos sugieren que la planificación de la gestión internacional a través de la estructuración departamental guarda relación con la forma en que se divide el trabajo y se establecen mecanismos de coordinación en una empresa (Stanton, Etzel y Walker, 2000; Rheingold, 2004). Dicha morfología no es ni mejor ni peor que otra, pero la facilita el desarrollo de la estrategia de exportación (Aulakh y Kotabe, 1997). Asimismo, Cateora y Graham (2006) señalan que aquellas empresas que presentan mayores grados de formalidad en la manera de organizarse representan un atractivo foco de interés para atraer a sus filas al personal más preparado y capacitado para dirigir la gestión internacional. Así pues, y atendiendo a la argumentación anterior, formulamos la siguiente hipótesis:

\section{H5: Las empresas que enfatizan en la planificación de su gestión internacional pre- sentan un mayor grado de internacionalización}

La estrategia competitiva distingue entre los procesos de formulación y los procesos de implementación. Atendiendo a los primeros, - los procesos de formulación -, cabe señalar que tanto la selección de mercados como la decisión de la forma de entrada a los mercados previamente seleccionados son dos aspectos determinantes para que el proceso de expansión internacional tenga éxito (Reid, 1981; Erramilli, 1993; Luo, 2001, entre otros). Para acometer adecuadamente ambos aspectos, surge la necesidad de detentar niveles de información -en cantidad y calidad- adecuados. Con esto se alude directamente al proceso de inteligencia de mercado, fundamental para el desarrollo internacional de la empresa (Shane, 1996; Buckley y Casson, 1998; Aurifeille, 2002; Brouthers y Brouthers, 2003; Czinkota y Ronkainen, 2004). Las alternativas varían entre concentrarse en una pequeña cantidad de destinos o por el contrario, estar presente en una mayor cantidad de mercados.

Algunos autores respaldan la idea de que concentrarse en un menor número de mercados favorece la obtención de mayores cuotas de mercado con una mayor rentabilidad en el 
tiempo (por ejemplo, Bodur, 1994). Por el contrario, otros trabajos defienden la puesta en práctica de un proceso de expansión internacional más dinámico que le permita a la empresa desarrollar sus actividades en un mayor número de mercados geográficos (Czinkota y Ursic, 1991; Alonso y Donoso, 1998; Aulakh, Kotabe y Teegen 2000). En cualquier caso, tanto el proceso de recogida de información como su posterior análisis, son actividades básicas en la toma de decisiones. Por ello, no es poca la literatura que señala la necesidad por parte de las empresas de mantener un sistema de información de marketing multinacional (SIMM), permanente, vigente y actualizado (Cateora, 1996). Desde esta perspectiva, proponemos la siguiente hipótesis:

\section{H6: Las empresas que enfatizan en la evaluación y selección de mercados internacio- nales, presentan un mayor grado de internacionalización}

La relevancia del marketing internacional se ha ido manifestando con mayor intensidad en la medida que el flujo de negocios y transacciones transfronterizas se ha ido incrementado en los últimos veinte años (Rheingold, 2004; Cateora y Graham, 2006). En este sentido, hay que señalar que las variables referidas al marketing internacional han sido tradicionalmente asociadas al grado de internacionalización de las empresas. Sin embargo, las conclusiones a este respecto no han sido unívocas (Aaby y Slater, 1989; Morgan, 1992; Albaum, Strandskov y Duerr, 1998; Wilson y Huang, 2003, entre otros).

Uno de los aspectos que ha generado mayor debate está relacionado con el producto (la oferta) y la decisión de adoptar una estrategia de estandarización versus decantarse por la adaptación a las especificidades del mercado demandante (Shoham, 1999). A este respecto, es preciso indicar que si bien la actuación en mercados globales por parte de una empresa determinaría una decisión en favor de estrategias de mayor estandarización de productos, las diferencias en las necesidades de los clientes inclinarían la decisión hacia modificaciones y adaptaciones de las características del producto. Por ello, algunos autores defienden la intensidad y magnitud de los fenómenos culturales, sociales, demográficos, etc. en los mercados de destino como aspecto determinante en la decisión sobre la conveniencia de seguir una estrategia de adaptación versus estandarización (Nieto, Llamazares y Cerviño, 1997; Czinkota y Ronkainen, 2004; Peter y Olson, 2006).

Este aspecto resulta de particular importancia dado que la adaptación de cualquier producto o servicio a las necesidades del mercado demandante necesariamente presionará la estructura de costes de la empresa, representando una desventaja respecto del producto no modificado. A este respecto algunos autores sugieren que la estrategia «transnacional» ${ }^{8} \mathrm{se}$ exime de la necesidad extrema de adaptación, apelando al fenómeno de la globalización. Por el contrario, el criterio de adaptación masiva ofrece una salida a la dicotomía anterior, al permitir mantener en el país de origen la totalidad de los procesos que requieren de un mayor grado de estandarización y acercar los procesos de producción que requieren mayor grado de diferenciación a los países de destino (Pepper y Rogers, 2000; Pine II y Gillmore, 2000; Kaplan, Schoder y Haenlein, 2007). Atendiendo a los argumentos anteriores, formulamos la siguiente hipótesis:

\footnotetext{
${ }^{8}$ Mc Donalds, Sony yToyota, son algunos casos de empresas exitosas que han quebrado la dicotomía de adaptación vs. estandarización por medio de una estrategia de corte transnacional. (Stanton, 2000 y Cateora, 2006).
} 


\section{H7: Las empresas que enfatizan en la adaptación de su estrategia de marketing inter- nacional presentan un mayor grado de internacionalización.}

En resumen, los autores proponen un modelo de análisis en el cual el grado de internacionalización se puede ver afectado por las siguientes variables explicativas: i)el tamaño de la empresa; ii) la gestión de calidad; iii) las tecnologías de la información y la comunicación; iv) la planificación de la gestión internacional; v) la capacitación y formación de los recursos humanos; vi) la evaluación y selección de los mercados internacionales; y finalmente, vii) la adaptación de la estrategia de marketing internacional llevada a cabo por la empresa, tal y como se resume en la Figura 1.

\section{METODOLOGÍA}

\subsection{Medición de la Variable Dependiente}

El grado de internacionalización (INTER) fue medido a través de la elaboración de un índice de valor compuesto, multidimensional, dinámico y graduado conforme a indicadores de gestión alineados con la realidad particular de la industria vitivinícola chilena. En consecuencia, la variable dependiente considerada en este trabajo es resultado de la elaboración de un promedio ponderado de las siguientes variables referidas al periodo 2004-20079.

1) Volumen de exportación (VE): Registra el valor monetario promedio neto de las exportaciones FOB registradas en dólares americanos por las empresas vitivinícolas durante el periodo de análisis.

2) Precio promedio unitario (PP): Registra el valor del precio promedio de cada litro de vino exportado por las empresas vitivinícolas.

3) Continuidad Exportadora (CE): Mide la estabilidad de las empresas vitivinícolas en el negocio exportador, según la cantidad de años que cuenta con registro de exportaciones.

4) Dinamismo exportador (DE): Mide el crecimiento del volumen de exportaciones de las empresas vitivinícolas entre el primer y último periodo en el que se registraron exportaciones.

5) Dinamismo del precio promedio unitario (PE): Mide el crecimiento del precio promedio de cada litro exportado entre el primer y último periodo en el que se registraron exportaciones.

6) Dinamismo exportador sostenido (DS): Mide la estabilidad del crecimiento de las exportaciones de las empresas vitivinícolas según el número de veces que registraron tasa de crecimiento positivas durante el periodo analizado

\footnotetext{
${ }^{9}$ Cabe señalar que un mecanismo de similar estructuración para medir el desempeño exportador fue anteriormente desarrollado por los autores Morí-Koenig, Yoguel, Milesi y Gutiérrez en el trabajo «Buenas Prácticas de las PYME Exportadoras Exitosas», así como en el trabajo «Estudio comparado sobre el éxito exportador PYME en Argentina, Chile y Colombia», en el año 2007.
} 
De acuerdo a lo anterior, la función utilizada para el cálculo de la variable dependiente presenta la siguiente expresión:

INTER $=\left(\mathrm{VE}^{*} \mathbf{0 , 3 5}\right)+(\mathrm{PP} * 0,20)+(\mathrm{CE} * 0,10)+(\mathrm{DE} * 0,15)+(\mathrm{PE} * 0,15)+(\mathrm{DS} * 0,05)$

Tal y como se puede observar, INTER otorga un mayor peso al volumen exportado ( $\mathrm{VE}=0,35)$, seguido del precio promedio por litro $(\mathrm{PP}=0,20)$, el dinamismo exportador $(\mathrm{DE}=0,15)$ y del precio promedio $(\mathrm{PE}=0,15)$. Finalmente se ponderó la continuidad exportadora $(\mathrm{CE}=0,10)$ y el indicador de dinamismo exportador sostenido $(\mathrm{DS}=0,05)$ con un peso menor que el resto por considerarse que dichos componentes contienen un carácter más bien complementario en la ecuación ${ }^{10}$. En este sentido, es preciso señalar que el indicador INTER tomó en cuenta tres grandes aspectos de la trayectoria de las empresas vitivinícolas durante el periodo. En primer lugar, se consideraron los dos indicadores de gestión más utilizados por la industria vitivinícola nacional: el flujo al exterior (volumen total de exportación neta) y el precio por unidad comercializada en el exterior (precio promedio por litro). En segundo término, se consideró la permanencia en los mercados externos a través de la variable continuidad, la cual mide el total de años durante los cuales la empresa efectivamente exportó. Seguidamente, se incorporó el dinamismo exportador para reflejar la tasa de cambio que experimenta tanto por el volumen exportado como el precio promedio por litro de vino al cual se exportó durante el periodo de análisis, 2004 y 2007 respectivamente. Finalmente y de forma complementaria, se tuvo en cuenta el dinamismo sostenido correspondiente al análisis del signo que toma la tasa de cambio del volumen exportado durante el periodo de estudio.

\subsection{Medición de las Variables Independientes y Recogida de Información}

Para definir la muestra se tomó como ámbito todo el territorio nacional chileno y como universo poblacional al conjunto de las 231 empresas vitivinícolas que estaban registradas como exportadoras durante el año 2007 en la base de datos de la dirección de promoción de exportaciones (PROCHILE). Con el objeto de seleccionar las unidades concretas de análisis se procedió a través de la técnica de muestreo aleatorio estratificado. El objetivo de este método permitió optimizar el diseño de la muestra considerando el criterio de estratificación por grado de internacionalización. La selección inicial de la muestra de 209 empresas nos permitió trabajar con un error muestral del 2,0\%. No obstante, dicho error se vio incrementado ex-post ya que se obtuvieron un total de 87 cuestionarios en el final del proceso. En este sentido es preciso señalar que si bien el total de cuestionarios respondidos

\footnotetext{
${ }^{10}$ El valor de las ponderaciones fue determinado a través de los resultados obtenidos en el juicio de expertos realizado con varios miembros de la industria vitivinícola chilena a fin de calibrar las variables seleccionadas de acuerdo con la realidad propia del sector. El trabajo consideró inicialmente la formulación de los criterios así como la revisión gradual de cada uno de los factores seleccionados para luego generar la determinación del peso relativo de cada uno de estos a partir de un análisis de consenso. En este sentido, es preciso indicar que en el análisis Delphi (Aaker y Day, 2003) llevado a cabo participaron dirigentes gremiales de la industria vitivinícola chilena, gerentes y directores de empresas vitivinícolas, analistas del sector, así como académicos relacionados con el tema.
} 
representó un porcentaje del $37 \%$ respecto del total de empresas del sector, el error muestral ex-post estuvo situado en torno al $8 \%$ con un grado de confianza al 95,5\% ${ }^{11}$. Dicho proceso permitió la generación de dos segmentos distintos y por ello, se llevó a cabo una subestratificación de acuerdo con el indicador del tamaño de empresa. El objetivo de este segundo paso fue definir colectivos de empresas lo más homogéneos posibles respecto de las variables que se pretendían estudiar, así como maximizar la fiabilidad de las estimaciones para un tamaño muestral fijo. Los sub-grupos resultantes corresponden a grandes empresas con alto desempeño exportador, medianas empresas con alto desempeño exportador, pequeñas empresas con alto desempeño exportador, grandes empresas con bajo desempeño exportador, medianas empresas con bajo desempeño exportador y pequeñas empresas con bajo desempeño exportador.

Para la contrastación de las siete hipótesis formuladas en este trabajo, se procedió a la recogida de información por medio de cuestionarios con escalas de Likert repartidos a las empresas vitivinícolas. La selección de las empresas a encuestar se realizó mediante muestreo aleatorio simple en cada uno de los sub-grupos identificados anteriormente, sin establecer ningún criterio particular de asignación geográfica, ya que se buscaba la máxima representatividad para el conjunto del territorio nacional. Con el objeto de validar las preguntas del cuestionario, se efectuó un pre-test con profesionales pertenecientes a la industria vitivinícola para detectar la existencia de posibles errores y limitaciones que pudieran estar presentes antes de la recogida de la información. Seguidamente, el objetivo se situó en la consecución del mayor número posible de cuestionarios válidos completados ya que el perfil del encuestado (principalmente dueños y propietarios de empresas vitivinícolas medianas y pequeñas de corte familiar), suele mostrarse poco proclive a la entrega de información específica, veraz y objetiva sobre experiencias anteriores. Con el fin de superar este obstáculo, los autores contactaron con Wines of Chile para que les proporcionara su colaboración. La respuesta de dicho organismo fue favorable y su Dirección elaboró una carta en la que resaltaba la necesidad de este estudio y solicitaba la colaboración de las empresas receptoras de la misma. Esta carta fue adjuntada como portada del cuestionario.

Con relación al cuestionario, es preciso señalar que éste fue enviado por e-mail a empresas vitivinícolas exportadoras de Chile que figuraban en los registros de exportación a diciembre de 2007. Específicamente el envío fue dirigido al gerente de exportaciones de las empresas y en ausencia de dicho cargo, al gerente comercial, gerente general o representante legal registrado en las distintas bases de datos utilizadas. En este sentido, es preciso mencionar que la persona encargada de recibir y contestar el cuestionario fue el responsable en materia de gestión internacional de cada empresa. En el caso de las grandes empresas vitivinícolas chilenas (17\% de la muestra) que contaban con estructura organizacional pertinente, el gerente de gestión internacional o de exportaciones fue quién respondió a las encuestas. Por otra parte, en el caso de las pequeñas y medianas empresas vitivinícolas ( $83 \%$ de la muestra), _-normalmente carentes de estructura organizacional —,

\footnotetext{
${ }^{11}$ De acuerdo a Eusebio y Llonch (2006), este valor es bastante usual en investigaciones realizadas en España e Italia que trabajan con un error sectorial en torno al 10\%. Por otra parte, la comparación de la distribución de las empresas por tramos de tamaño proporcionales, nos permitió aceptar la representatividad de las respuestas obtenidas ya que con respecto al sesgo de no-respuesta, los resultados mostraron que las empresas que no contestaron el cuestionario se distribuyeron de forma similar al universo de partida.
} 
el responsable en materia de gestión internacional casi siempre era ser el gerente general o representante legal de la empresa y por tanto, resultó ser quien efectivamente respondió el cuestionario.

Como consecuencia del primer envío se obtuvieron 48 cuestionarios válidos. Posteriormente se hizo un segundo envío exclusivamente a aquella parte de la base que no respondió la encuesta en el primer envío, alcanzándose un total de 39 cuestionarios. La suma de ambos envíos generó un total de 87 encuestas completadas, lo que representó un $36 \%$ de las empresas del sector. Cabe señalar que en la $2^{\text {a }}$ etapa se utilizó apoyo telefónico y apoyo en terreno con el fin de facilitar la recepción de los cuestionarios que no fueron devueltos a través del correo electrónico. La Tabla 2 recoge la operativización de las variables independientes del modelo ${ }^{12}$.

Tabla 2

\section{Operativización de las Variables Independientes del Modelo}

\begin{tabular}{|lcc|}
\hline Variable independientes & Código & $\begin{array}{c}\text { Rango } \\
\text { respuesta }\end{array}$ \\
\hline Tamaño de la empresa & TAM & Rango (1-3) \\
Gestión en aseguramiento de la calidad & QUA & Rango (1-5) \\
Gestión en uso de TIC & TIC & Rango (1-5) \\
Planificación de la gestión internacional & PLA & Rango (1-5) \\
Formación y capacitación del recurso humano involucrado en las actividades de & RHU & Rango (1-5) \\
gestión internacional & EVA & Rango (1-5) \\
Evaluación y ánalisis de los mercados internacionales & MKT & Rango (1-5) \\
Flexibilidad y adaptación de la estrategia de marketing internacional & &
\end{tabular}

\subsection{Contraste empírico}

El método de análisis de regresión múltiple por medio de mínimos cuadrados ordinarios permitió determinar la relación existente entre las variables utilizadas a partir de la muestra con la que se trabajó. Atendiendo a los resultados recogidos en la Tabla 3, la ecuación y sus coeficientes adquirieron la siguiente expresión:

\footnotetext{
${ }^{12}$ De acuerdo con la tabla 2, en el caso de la variable TAM el rango posible de valores factibles se mueve entre 1 y 3, donde: (1) implica que la empresa factura sobre MMU\$ 5 y tiene más 200 empleados se denomina Gran Empresa (GE); (2) agrupa cualquier caso distinto al anterior pero con facturación > MMU\$ 1 y por ello, se le denomina PYME; finalmente, (3) implica que la empresa factura menos de MMU\$ 1, por lo que se le denomina pequeña empresa (PYPE). Criterio: Corporación de fomento de chile (CORFO Chile). En el caso de las variables; QUA, TIC, PLA, RHU, EVA y MKT, el rango posible de valores está asociado a una escala de Likert entre 1 y 5 . Para ello, se requirió que el entrevistado indicara su grado de acuerdo o desacuerdo con respecto a una variedad de afirmaciones relacionadas con el objeto de las temáticas planteadas permitiéndole registrar en cada una de ellas el grado de énfasis con el cual mejor relación de percepción lograba establecer en cada uno de los temas (AAker y G. Day, 2003).
} 
INTER $=-8.05+3.37 * \mathrm{EVA}+5.64 * \mathrm{MKT}+2.51 * \mathrm{PLA}+3.40 * \mathrm{QUA}+1.99 * \mathrm{RHU}+0.36 * \mathrm{TAM}+1.74 * \mathrm{TI}^{\circ}$

Tabla 3

Resultados del Primer Modelo de Regresión

\begin{tabular}{|crlll|}
\hline \hline Variable & Coefficient & Std. Error & t-Statistic & Prob. \\
\hline \hline C & -8.059258 & 2.839661 & -2.838106 & 0.0058 \\
MVA & 3.372675 & 0.939207 & 3.590983 & 0.0006 \\
PLA & 5.646291 & 1.105010 & 5.109719 & 0.0000 \\
QUA & 2.516142 & 0.961129 & 2.617902 & 0.0106 \\
RHU & 3.400006 & 0.635583 & 5.349432 & 0.0000 \\
TAM & 1.994902 & 0.944998 & 2.111012 & 0.0379 \\
TIC & 0.366302 & 1.255936 & 0.291657 & 0.7713 \\
& 1.748710 & 0.830009 & 2.106858 & 0.0383 \\
R-squared & 0.864260 & Mean dependent var & 45.36782 \\
Adjusted R-squared & 0.852233 & S.D. dependent var & 18.79579 \\
S.E. of regression & 7.225196 & Akaike info criterion & 6.880474 \\
Sum squared resid & 4124.073 & Schwarz criterion & 7.107224 \\
Log likelihood & -291.3006 & F-statistic & 71.85665 \\
Durbin-Watson stat & 1.820687 & Prob(F-statistic) & 0.000000 \\
\hline \hline
\end{tabular}

Tal y como se puede observar, la bondad del ajuste del modelo ofrece una capacidad explicativa en torno al $86 \%$. Así pues, el valor asociado a los coeficientes de determinación $\mathrm{R}^{2}$ y $\mathrm{R}^{2}$ ajustado fue del $86,4 \%$ y $85,4 \%$, respectivamente. Asimismo, el test de significatividad conjunta del modelo obtuvo un valor de contraste $\mathrm{F}=71,8$ resultando significativo a un nivel 0,00 . Por otra parte los resultados de los estadísticos y sus probabilidades muestran que todas las variables independientes fueron significativas con la excepción del tamaño de la empresa (TAM). Ello nos permite aceptar a un nivel 0,00 las hipótesis asociadas a la utilización de estándares internacionales de gestión de calidad (H2), evaluación y selección de mercados (H6), así como la adaptación de la estrategia de marketing internacional (H7). Por el contrario, las hipótesis vinculadas a la utilización de las técnicas de la información y comunicación (H3), formación de personal (H4) y planificación de la gestión internacional (H5) fueron aceptadas a un nivel de confianza del 95 por ciento.

A partir de estos resultados, los autores consideraron oportuno verificar la posible «redundancia» de la variable tamaño (TAM). Para ello se utilizó el test denominado «Redundant Variables-Likelihood Ratio». Los datos obtenidos concluyeron la imposibilidad de rechazar la hipótesis nula del contraste de «redundancia». Por lo tanto, y debido a que la probabilidad de equivocarse resultó muy alta $(75,9 \%)$ se tuvo que aceptar que la variable (TAM) era efectivamente una variable redundante. Adicionalmente se pudo observar que el coeficiente de determinación ajustado de este modelo ( $\mathrm{R}^{2}$ ajustado) fue superior al del modelo original lo que ratifica que no se está omitiendo ninguna variable relevante al excluir a la variable (TAM). Por el contrario, la capacidad explicativa del nuevo modelo mejoró respecto del anterior. Los resultados se recogen en la Tabla 4. 
Tabla 4

Resultados del Redundant Variables-Likelihood Ratio

\begin{tabular}{|llll|}
\hline \multicolumn{2}{l}{ Redundant Variables: TAM } & & \\
\hline \hline F-statistic & 0.085064 & Probability & 0.771313 \\
Log likelihood ratio & 0.093627 & Probability & 0.759615 \\
\hline
\end{tabular}

A raíz de los resultados obtenidos en el análisis de redundancia antes comentado, se procedió a eliminar la variable TAM del modelo explicativo, adquiriendo la siguiente expresión:

INTER $=-7.92+3.43 * \mathrm{EVA}+5.67 * \mathrm{MKT}+2.55 * \mathrm{PLA}+3.42 * \mathrm{QUA}+2.08 * \mathrm{RHU}+1.71 * \mathrm{TIC}$

Tabla 5

Resultados del Segundo Modelo de Regresión

\begin{tabular}{|crlrl|}
\hline \hline Variable & Coefficient & Std. Error & t-Statistic & Prob. \\
\hline \hline C & -7.922592 & 2.784671 & -2.845073 & 0.0056 \\
EVA & 3.432988 & 0.910904 & 3.768771 & 0.0003 \\
PKT & 5.677733 & 1.093432 & 5.192579 & 0.0000 \\
QUA & 2.550404 & 0.948452 & 2.689017 & 0.0087 \\
RHU & 3.422290 & 0.627355 & 5.455107 & 0.0000 \\
TIC & 2.082239 & 0.891158 & 2.336554 & 0.0220 \\
R-squared & 1.717690 & 0.818445 & 2.098723 & 0.0390 \\
Adjusted R-squared & 0.864114 & Mean dependent var & 45.36782 \\
S.E. of regression & 7.1839761 & S.D. dependent var & 18.79579 \\
Sum squared resid & 4128.514 & Schwarz criterion & 7.056968 \\
Log likelihood & -291.3474 & F-statistic & 84.78828 \\
Durbin-WVatson stat & 1.826032 & Prob(F-statistic) & 0.000000 \\
\hline \hline
\end{tabular}

Tabla 6

Matriz de Correlaciones

\begin{tabular}{|c|c|c|c|c|c|c|}
\hline \multicolumn{9}{|c|}{ Correlation Matrix } \\
\hline \hline & MKT & EVA & PLA & QUA & RHU & TIC \\
\hline MKT & 1.000000 & 0.648506 & 0.659595 & 0.242026 & 0.605168 & 0.513296 \\
\hline EVA & 0.648506 & 1.000000 & 0.540489 & 0.414674 & 0.428072 & 0.482811 \\
\hline PLA & 0.659595 & 0.540489 & 1.000000 & 0.314867 & 0.374178 & 0.459426 \\
\hline QUA & 0.242026 & 0.414674 & 0.314867 & 1.000000 & 0.317819 & 0.310459 \\
\hline RHU & 0.605168 & 0.428872 & 0.374178 & 0.317819 & 1.0010100 & 0.389575 \\
\hline TIC & 0.513296 & 0.482811 & 0.459426 & 0.310459 & 0.389575 & 1.000000 \\
\hline & & & & & & \\
\hline
\end{tabular}


De este modo, el nuevo modelo adquiere un valor de contraste $\mathrm{F}=84,7$, resultando significativo a un nivel del 0.000 . Por otra parte los resultados de los estadísticos t y sus pvalores asociados ratificaron la significatividad estadística de la relación entre la variable dependiente (INTER) y las 6 variables independientes propuestas. En concreto, con la excepción de la formación de personal (RHU) y las técnicas de la información y la comunicación (TIC), todas resultaron significativas a un nivel de confianza del 99 por ciento. Esto nos permitió confirmar las hipótesis H2, H5, H6 y H7 a un nivel del 0,00. Por el contrario, las hipótesis $\mathrm{H} 3$ y $\mathrm{H} 4$ fueron confirmadas a un nivel del 0,05.

Para la detección de la heteroscedasticidad en el nuevo modelo, se utilizó el test de White. A este respecto, se sabe que bajo la hipótesis nula el estadístico de White se distribuye como una chi-cuadrado con r-1 grados de libertad. Por lo tanto si el número de observaciones multiplicado por el $\mathrm{R}^{2}$ de la regresión «auxiliar» se distribuye como una chicuadrado de 6 grados de libertad, (el número total de regresores sin tomar en cuenta el término constante de la regresión auxiliar), se obtiene que Obs. * R-cuadrado = 12,32 < 12,59, lo que permitió rechazar la hipótesis nula de ausencia de heteroscedasticidad. Por otra parte, para llevar a cabo la detección de presencia de autocorrelación en los términos de error, se utilizó el test de Durbin-Watson. Tal y como se recoge en la Tabla 5, se obtuvo un valor de contraste de 1,826 , en tanto que los puntos de significatividad para $d L$ y $d U$ para las 87 observaciones de la regresión y los 6 regresores utilizados en el nuevo modelo (a un nivel de confianza del 95 por ciento) fueron de: $(d L: 1,503)$ y $(d U: 1,801)$ respectivamente. Lo anterior permitió rechazar la existencia de autocorrelación al confirmarse que el contraste cumple con la siguiente regla de decisión: $d U<d<4-d U$ (Gujarati, 2006, pp.430-434, entre otros). Finalmente, es preciso señalar que los resultados de las pruebas de multicolinealidad efectuadas permitieron descartar en forma definitiva la presencia de multicolinealidad perfecta así como la presencia de una intensa correlación entre las variables ${ }^{13}$.

\section{CONCLUSIONES, CONTRIBUCIONES Y FUTURAS LINEAS DE INVESTI- GACIÓN}

Atendiendo al análisis de los contrastes realizados, los resultados indican que todas las variables independientes propuestas en este trabajo - con excepción del tamaño de empresa (TAM) - , guardan una relación positiva y estadísticamente significativa con la mayor o menor presencia de las empresas vitivinícolas en el extranjero. La escasa significación estadística observada entre el tamaño de la empresa (TAM) y la variable dependiente (INTER) se debe a varios motivos. En primer lugar, las empresas vitivinícolas aparecen cada vez más focalizadas en favorecer sustitución de mano de obra por tecnología y procedi-

\footnotetext{
${ }^{13}$ Con el propósito de analizar una eventual colinealidad se obtuvo la matriz de correlación no evidenciándose una fuerte relación entre las variables pareadas. Por otra parte, los indicadores de tolerancia $\left(1-\mathrm{R}^{2}\right)$ del modelo de regresión, se enmarcaron entre los valores extremos de 0,63 para la variable TIC y 0,25 para la variable MKT, con indicadores FIV que oscilaron entre 1,59 y 4,0 respectivamente. Finalmente se exploró el comportamiento de un potencial nuevo modelo de regresión a partir de la eventual eliminación de la variable MKT (la variable extrema de la ecuación), logrando corroborarse la relevancia de la misma para el modelo original al constarse que el nuevo coeficiente de determinación ajustado reducía significativamente la capacidad explicativa en la nueva especificación.
} 
mientos de gestión, por lo que la cantidad de personal ha dejado paulatinamente de ser un aspecto explicativo central en la definición de su estrategia internacional. Por otra parte, el criterio utilizado en esta investigación para diferenciar por tamaño, consideró no solo la dotación de personal, sino también la facturación total de cada empresa, la cual a todas luces constituye una redundancia evidente por cuanto esta incluye la facturación tras frontera. Por ello, tal y como se explicó en la sección anterior, los autores optaron por eliminar dicha variable del modelo explicativo final.

Junto a ello y con respecto a las capacidades competitivas que podrían explicar el grado de internacionalización de las empresas pertenecientes al sector vitivinícola chileno, se eligió el factor «gestión de calidad» (QUA). En este sentido, los resultados obtenidos nos permiten afirmar que las empresas vitivinícolas con mayor presencia internacional son aquellas que habiendo internalizado la importancia de la calidad, han asumido el compromiso de organizar su gestión bajo las normas y procedimientos que ofrecen la certificación de normas internacionales del tipo ISO, APPCC, o equivalente. Por otra parte, los resultados destacan la importancia de las capacidades tecnológicas aludiendo directamente al concepto de «tecnologías de la información y la comunicación» (TIC). A este respecto, se logró evidenciar que las empresas vitivinícolas chilenas que poseen y utilizan regularmente herramientas TIC, tales como web sites actualizados, portales de internet, sistemas de ventas on-line, show-room digitales, portales intranet corporativo, etc. logran favorecer la comunicación eficiente no sólo entre la propia empresa sino también con sus proveedores y clientes, lo cual favorece su expansión internacional.

Con relación a las variables relacionadas con la gestión internacional y la formación de los recursos humanos, cabe señalar que el efecto de ambas variables sobre la variable dependiente resultó significativo a un nivel de confianza del 99 y 95 por ciento, respectivamente. Este trabajo demuestra que las empresas que cuentan con una metodología apropiada para desarrollar el proceso de planificación de la gestión internacional a través de normas y procedimientos acerca de la forma de actuar en materia de exportaciones, tienden a presentar un grado de internacionalización mayor. Asimismo, respecto a la evaluación y selección de los mercados internacionales así como la estrategia de marketing internacional, ambos regresores ofrecieron significatividad suficiente como para aceptar las hipótesis respectivas, sin embargo el análisis de la regresión ratificó la mayor importancia relativa de la variable (MKT). Este resultado parece confirmar que las empresas vitivinícolas chilenas que evidencian un mayor grado de internacionalización, cuentan con sistemas formales de información de marketing e investigación de mercados. Sin embargo, las evidencias obtenidas parecen indicar que durante el proceso de la evaluación y formulación de la estrategia, a veces prevalece la «la experiencia y el olfato empresarial de los directivos» sobre cualquier consideración metodológica formal realizada por expertos.

Dado que los autores no tienen constancia de la existencia de algún estudio anterior - conceptual y/o empírico - orientado a la búsqueda de los factores que determinan las diferencias del grado de internacionalización de las empresas vitivinícolas, la presente investigación tuvo como objetivo cubrir este vacío. Para ello, se elaboró un modelo que permitiera entender y predecir la estrategia de expansión internacional llevada a cabo por las empresas pertenecientes a la industria vitivinícola chilena, un sector de altísima relevancia para dicho país pero con escasa aproximación académica en materia de gestión internacional. Además, este artículo incluye la elaboración y contrastación empírica de un indicador 
del grado de internacionalización construido a partir de un índice de valor compuesto, multidimensional y dinámico, calibrado conforme al perfil de la industria vitivinícola chilena, contexto sobre el cual se realizó este estudio. Precisamente, creemos que una de las principales contribuciones a la literatura dimana de este hecho. Sin embargo, la contribución de este estudio no sólo es científica sino también práctica. Esto se debe a que tanto la elaboración de una base de datos generada sobre la base de empresas exportadoras vitivinícolas chilenas entre los años 2004 y 2007, como el análisis de los resultados obtenidos, constituyen recursos potencialmente capitalizables por parte de empresarios vitivinícolas y/o agencias estatales involucradas en todo el proceso exportador de la industria.

Una de las principales limitaciones de este trabajo es que la muestra sólo incluye empresas pertenecientes a la industria vitivinícola chilena. En este sentido, y como futura línea de investigación, proponemos que futuros trabajos analicen el grado de internacionalización de empresas de nacionalidad no chilena y comparen los resultados obtenidos. Por otra parte, proponemos que se analicen variables complementarias a las propuestas en este estudio para explicar el proceso de expansión internacional. Por último, sería interesante analizar la relación entre el «performance» de la empresa (rentabilidad económica, ventas, cuota de mercado, etc.) y grado de internacionalización y por ello, lo proponemos como futura línea de investigación.

\section{REFERENCIAS BIBLIOGRÁFICAS}

AABY, N. Y SLATER, E. (1989): «Management Influence on Export Performance: A review of the Empirical Literature 1978-1988», International Marketing Review, Vol. 6, No. 4, pp. 7-22.

AAKER, D. Y DAY, G. (2003): Investigación de Mercados, séptima edición, Mc Graw Hill, México. ALBAUM, G. STRANDSKOV, J. Y DUERR, E. (1998): International Marketing and Export Management, Harlow, UK, Addison Wesley.

ALDERETE, V. (2007): «Nuevas tecnologías de la información y de la comunicación: factores explicativos de la conducta exportadora en Argentina», Cuadernos de Docencia Universidad Nacional Del Sur, (Marzo).

ALONSO, J. A. Y V. DONOSO (1998): Competir en el exterior. La empresa española y los mercados internacionales, ICEX, Madrid.

ALONSO, J. A. Y V. DONOSO (2000): Modelización del comportamiento de la empresa exportadora española, ICEX, Madrid.

ALFREDO, E. (2001): Administración de los Recursos Humanos, Valletta Ediciones, Buenos Aires.

ANDERSON, S., DALY, D. Y JOHNSON, M. (1999): «Why Firms Seek ISO 9000 Certification: Regulatory Compliance or Competitive Advantage?», Production and Operation Management, Vol. 8, pp. 28.43.

AULAKH, P. Y KOTABE, M. (1997): «Performance Implications of Channel Integration in Foreign Markets», Journal of International Business Studies, Vol. 28, No. 1, pp. 145-175.

AULAKH, P. KOTABE, M. Y TEEGEN, H. (2000): «Export Strategies and Performance of Firms from Emerging Economies: Evidence from Brazil, Chile, and Mexico», Academy of Management Journal, Vol. 43, No. 3, pp. 342-361.

AURIFEILLE, J (2002): «Global vs. international involvement-based segmentation: A cross-national exploratory study», International Marketing Review. Vol. 19, No. 4, pp. 369-386.

BERNARD, A. Y JENSEN, J. (2004): «Why do Some Firms Export?», The Review of Economics and Statistics, Vol. 86, pp. 561-569. 
BLACK, S. Y MANDENHALL, M. (1991): A practical theory-based framework for selecting cross-cultural training methods, International Human Resources Management, eds. (Boston, Massachusetts).

BERNAL, E. Y PAJARES, E. (2006): «Las TIC como instrumento de internacionalización: el caso de la industria española», Economía Industrial, Vol. 361, pp. 111-120

BODUR, M. (1994): «Foreign Market Indicators, Structural Resources and Marketing Strategies as Determinants of Export Performance», Advances in International Marketing, Vol. 6, pp. 183-205.

BONACCORSI, A. (1992): «On the relationship between firm size and export intensity», Journal of International Business Studies, Vol. 23, No. 4, pp. 605-635.

BOHLANDER, G. W., SNELL, S. A. Y SHERMAN, A. W. (2001): Managing Human Resource, South-Western College Publishing, $12^{\mathrm{a}}$ edición, Cincinnati, Ohio.

BROUTHERS, K. Y L. BROUTHERS (2003): «Why Service and Manufacturing Entry Mode Choices Differ: The Influence of Transaction Cost Factors, Risk and Trust», Journal of Management Studies, Vol. 40, No. 5, pp. 1179-1203.

BUCKLEY, P. Y CASSON, M. (1998): «Analyzing Foreign Market Entry Strategies Extending the Internalization Approach», Journal of International Business Studies, Vol. 29, No. 3, pp. 539-562.

CALOF, J. (1993): «The Impact of Size on Internationalization», Journal of Small Business Management, Vol. 31, No. 4, pp. 60-69.

CALOF, J. Y VIVIERS, W. (1995): «Internationalization behaviour of small- and medium-sized South African enterprises», Journal of Small Business Management, No.Vol 33, No. 4, pp 71-79.

CALOF, J. (1994): «The Relationship between Firm Size and Export Behaviour Revisited», Journal of International Business Studies, Vol. 25, No. 2, pp. 367388.

CASTAGNINO, T. (2006), «Estándares internacionales de calidad y desempeño exportador: Evidencia a nivel firma», Revista del CEI Comercio Exterior e Integración, Argentina.

CATEORA, P. (1996): Marketing internacional, $8^{a}$ edición, Irwin, Madrid.

CATEORA, P. Y GRAHAM, J. (2006): Marketing internacional, 12 a edición, Mc Graw Hill, MéxiCO DF.

CERVIÑO, J. (2006): Marketing internacional: nuevas perspectivas para un mercado globalizado, Pirámide, Madrid.

CONTRACTOR, F. Y KUNDU, S. (1998a): «Franchising Versus Company-Run Operations: Modal Choice in the Multinational Hotel Sector», Journal of International Marketing, Vol. 6, No. 2, pp. 28-53.

CONTRACTOR, F. Y KUNDU, S. (1998b): «Modal Choice in a World of Alliances: Analyzing Organizational Forms in the International Hotel Sector», Journal of International Business Studies, Vol. 29 (Second Quarter), pp. 325-358.

CRICK, D. Y JONES, M. (2000): «Small high-technology firms and international and international high technology markets», Journal of International Marketing, Vol. 8(2), pp 63-85.

CZINKOTA, M. Y RONKAINEN, I. (2004): Marketing Internacional, séptima edición, Mc Graw Hill, Mexico.

CZINKOTA, M. Y URSIC, M. (1991): «Classification of Exporting Firms According to Sales and Growth into a Share Matrix», Journal of Business Research, Vol. 22, pp. 243-253.

DEAN, D., MENGUC, B. Y MYERS, C. (2000): «Revisiting Firms Characteristics, Strategy and Export Performance Relationship», Industrial Marketing Management, Vol. 29, pp. 461-477.

DEHNING, B. Y STRAPOULOS, T. (2003): «Determinants of Sustainable competitive advantage due to: an IT-unable strategy», The Journal of Strategic Information Systems, Vol. 12, No. 1, pp. 7-28.

DÍAZ-CHAO, A. (2003): «El efecto de las tecnologías digitales en la competitividad de la empresa española», Revista Estudios de Economía Aplicada, Vol.4, pp. 21-3.

DURAN, J. (1994): «Factores competitivos en los procesos de internacionalización de la empresa», Información Comercial Española., Vol. 735, pp. 21-41. 
ERRAMILLI, M. Y RAO, C. (1993): «Service firms’ international entry mode choice: a modified transaction-cost analysis approach», Journal of Marketing, Vol. 57, No. 5, pp. 19-38.

EUSEBIO, R. Y LLONCH, P. (2006): «Innovación Tecnológica y Resultado Exportador: Un análisis empírico aplicado al sector textil-confección español», Revista Europea de Dirección y Economía de la Empresa, Vol. 12, No. 2, pp. 73-88.

GARCÍA-CANAL, E., RIALP-CRIADO, A. Y RIALP-CRIADO, J. (2007): «Tecnologías de la información y comunicación (TIC) y crecimiento de la empresa», Información Comercial Es pañola, Vol. 838, pp. 125-145

HARRISON, L. Y HUNTINGTON, S. (2000): Culture Matters, Nueva York: Basic Books, «source: 1999 Harvard University symposium, Mass, USA.

JARILLO, J. (1996): Dirección Estratégica, $2^{\text {a }}$ edición, Mc Graw-Hill, Madrid.

JARILLO, J. Y MARTÍNEZ, J. (1991): Estrategia Internacional. Más allá de la exportación. Mc Graw-Hill, Madrid.

KAPLAN, A. SCHODER, D. Y HAENLEIN, M. (2007): «Factors influencing the adoption of mass customization: The impact of base category consumption frequency and need satisfaction», Journal of product innovation management, No. 24(2), pp. 101-116.

KIRPALANI, V. Y MACINTOSH, N. (1980): «International Marketing Effectiveness of Technology-Orientated Small Firms», Journal of International Business Studies, Winter: 81-89.

LABEAGA, J. Y MARTÍNEZ-ROS, E. (1994): «Estimación de un modelo de ecuaciones simultáneas con variables dependientes limitadas: Una aplicación con datos de la industria española», Investigaciones Económicas, XVIII (3), pp. 465-489.

LUO, Y. (2001): «Determinants of entry in an emerging economy: A multilevel approach», Journal of Management Studies, Vol. 38, No. 3, pp. 443-472.

LOANE, S. (2006): «The role of the internet in the internationalization of small and medium size companies», Journal of International Entrepreneurship, Vol. 3, pp 263-277.

MAUREL, C (2009): «Determinants of export performance in French wine SMEs», International Journal of Wine Business Research, Vol. 21, No. 2, pp. 118-141.

MELLE, M. Y RAIMOND, J. (2001): «Competitividad Internacional de las Pymes Industriales Españolas», XI congreso de ACEDE Zaragoza, 17-18 de septiembre.

MERINO, F. (1998): «La Salida al Exterior de la Pyme Manufacturera Española», Investigación comercial española, Vol. 773(Septiembre/Octubre)

MERINO, F. Y MORENO, L. (1996): «Actividad Comercial en el Exterior de las Empresas Manufactureras Españolas y Estrategias de Diferenciación de Productos», Papeles de economía española, Vol. 66, pp. 107-123.

MOORI-KOENIG, V. MILESI, D. Y YOGUEL, A. (2005) Perfil de la PyME exportadora exitosa. El caso colombiano, Ed: FUNDES, Colombia.

MOORI-KOENIG, V., MILESI, D. YOGUEL Y GUTIERREZ (2007) Buenas Prácticas de las PYME Exportadoras Exitosas, Ed: FUNDES, Colombia.

MORGAN, R (1992): «The International Marketing of Cyprus Wines: A Case Example, the UK Market», International Journal of Wine Marketing, Vol 4, No. 1, pp. 44-63.

NEELANKAVIL, J. MATHUR, A. Y ZANG, Y. (2000): «Determinants of managerial performance: Across-cultural comparison of the perceptions of middle-level managers in tour countries», Journal of International Business Studies, Vol. 31, No. 1, pp 121.140.

NIETO, M. J. (2006): «Las TIC como impulsoras de la internacionalización de la empresa», Claves de la Economía Mundial, pp. 70-78

NIETO, A. LLAMAZARES, O. CERVIÑO, J. (1997): «Marketing Internacional, Casos y Ejercicios Prácticos, Guía de soluciones y respuestas, Ediciones Pirámide, Madrid.

PAN, Y. Y TSE, D. K. (2000): «The hierarchical model of market entry modes», Journal of International Business Studies, Vol. 31, No. 4, pp. 535-554 
PANAYI, S. Y TRIGEORGIS, L. (1998): «Multi-stage real options: The cases of information technology infrastructure and international bank expansion», The Quarterly Review of Economics and Finance, Vol. 38, Special Issue, pp. 675-692.

PETER, P. Y OLSON, J. (2006): Comportamiento del Consumidor y Estrategia de Marketing. $7^{\mathrm{a}}$ Edición.

PINE II, J. Y GILMORE, J. (2000): Creating Customer-Unique Value through Mass Customization. Ed: Harvard Business School Press, Boston (Massachusetts).

PORTER, M. (1982). Estrategia competitiva. México: McGraw Hill.

REID, S. (1981): «The Decision-Maker and Export Entry and Expansion», Journal of International Business, Vol. 12, pp. 101-112.

REID, S. (1982): «The Impact of Size on Export Behavior in Small Firm», en M. R. Czinkota, Export Management: an International Context. Praeger Publish., NY. pp. 18-38.

RHEINGOLD, H. (2004): Multitudes Inteligentes: La próxima revolución Social, Editorial Gedisa.

ROBERTS, M. Y TYBOUT, J.R. (1997): «The Decision to Export in Colombia: an Empirical Model of Entry with Sunk Costs», American Economic Review, Vol. 87, pp. 545-564.

ROOT, F. (1994): «Entry Strategies for International Market». Lexington Book, Mc. Millan, Massachusetts.

SHANE, S. (1996): «Why franchise companies expand overseas», Journal of Business Venturing, Vol. 11, No. 2, pp. 73-88.

SHOHAM, A. (1999): «Bounded rationality, planning, standardization of international strategy, and export performance: A structural model examination», Strategic Management Journal, Vol. 9 , No. 1 , pp. 43-60.

STANTON, W. ETZEL, M. Y WALKER, B. (2000): Fundamentos de marketing, Mc Graw Hill, México.

STEVENS, N Y BURNS, E (2005): «Website Use in the Hawke's Bay Wine Region of New Zealand», International Journal of Wine Marketing. Vol. 17, No. 1, pp. 69-85.

WILSON, I. Y HUANG, Y (2003): «Wine Brand Naming in China», International Journal of Wine Marketing. Vol. 15, No. 3, pp. 52-63.

YOGUEL, G. NOVICK, M. MILESI, D. ROITTER, S Y BORELLO, J. (2004): «Información y conocimiento: la difusión de las tecnologías de información y comunicación en la industria manufacturera argentina», Revista CEPAL, Vol. 82.0 
ANEXO (ENCUESTA). (Ficha de respuestas)

\section{(A) Datos generales:}

Nombre de la viña

Valle vitivinícola asociado a la viña

Total de personas que trabajan en la viña

Total de países a los que exporto el último año

Relación (ventas totales/exportaciones) último año

\section{(B) Escala de percepción:}

\begin{tabular}{rrrrrr} 
Pregunta & NIVEL & NIVEL & NIVEL & NIVEL & NIVEL \\
/ Nivel & 1 & 2 & 3 & 4 & 5 \\
\hline
\end{tabular}

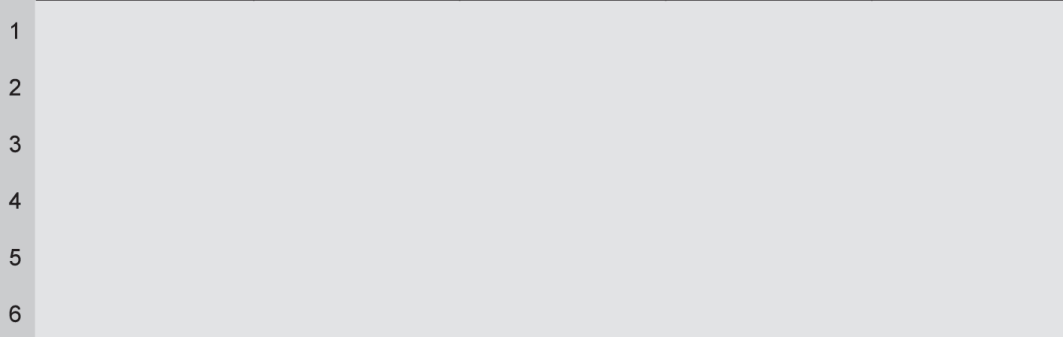

(C) Selección múltiples:

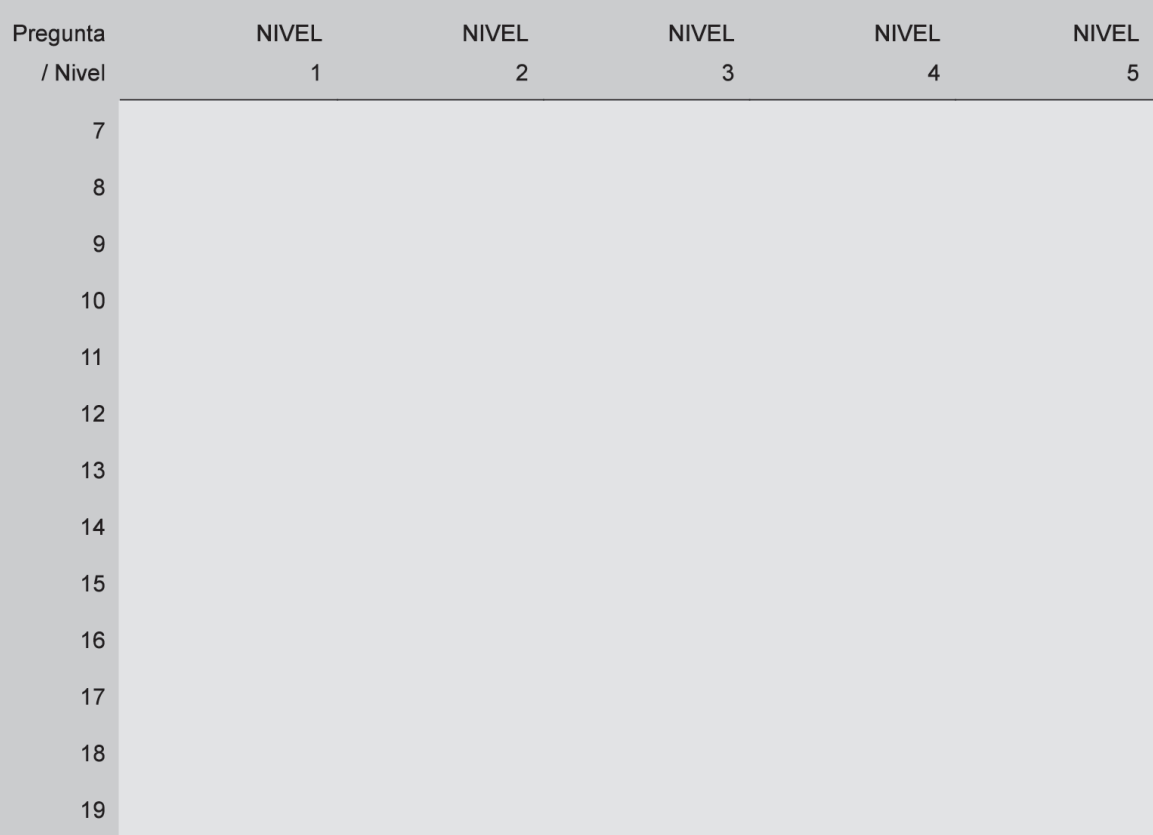




\section{(1) GESTION DE CALIDAD}

«La empresa enfatiza la utilización de procedimientos de aseguramiento de la calidad por medio de certificaciones en norma internacional del tipo: ISO, APPCC u otras equivalentes».

Máximo énfasis Mínimo énfasis

\begin{tabular}{|l|l|l|l|l|}
\hline & & & & \\
\hline
\end{tabular}

En relación con el tema de la referencia ¿Qué tipo de procedimientos concretos y herramientas específicas utiliza la empresa?

Criterios orientadores para ayudar a fijar el nivel de énfasis del factor

\section{NIVEL A:}

La empresa posee certificación en norma internacional (ISO, APPCC o equivalente) o está en preparación para obtenerla. En consecuencia, se ha internalizado la importancia de calidad y se ha asumido el compromiso de conducir la empresa bajo las normas y procedimientos correspondientes. Existe disciplina interna y estructuras de la gestión integral de calidad.

\section{NIVEL B:}

La empresa cuenta con una tradición de buen control de calidad pero no ha asumido el compromiso de una certificación a nivel ISO o equivalente. Sus productos han mantenido, a su juicio, un buen nivel de calidad dentro del mercado externo sin haber experimentado problemas significativos en este campo.

\section{NIVEL C:}

La empresa ha logrado exportar pese a haber experimentado algunos problemas en el aseguramiento de la calidad de sus productos.

NIVEL D:

La empresa no cuenta con procedimientos de aseguramiento de la calidad ni está en su plan tenerlos.

NIVEL E:

Ausencia del criterio. 


\section{(2) GESTION DE TECNOLOGIAS DE LA INFORMACION Y LA COMUNICA-} CIÓN (TIC)

«La empresa enfatiza la utilización de procedimientos y herramientas TIC, tales como por ejemplo: web sites interactivos (blogs), portales de Internet, sistemas de ventas on-line, show-room digitales, intranet corporativo u otros equivalentes».

\begin{tabular}{l} 
Máximo énfasis \\
\hline
\end{tabular}

En relación con el tema de la referencia ¿Qué tipo de procedimientos concretos y herramientas específicas utiliza la empresa?

Criterios orientadores para ayudar a fijar el nivel de énfasis del factor

NIVEL A:

La empresa posee herramientas TIC, tales como web sites actualizados, portales de internet, ventas on-line, show-room digitales, intranet corporativo, etc. los cuales son utilizados regularmente por el personal de la empresa y es asumido como gravitante en el proceso exportador a fin de favorecer la comunicación eficiente con proveedores, clientes y la comunidad en general.

NIVEL B:

La empresa cuenta con web-site y algunas herramientas TIC sin embargo no existe un compromiso periódico de actualización ni gran fluidez en el uso de dichas herramientas, por cuanto representa solo un complemento prescindible para la gestión exportadora de la empresa.

NIVEL C:

La empresa exporta con prescindencia casi absoluta del set de herramientas TIC no obstante estos existen al interior de esta.

NIVEL D:

La empresa no cuenta con set alguno de herramientas TIC para desarrollar su gestión exportadora.

NIVEL E:

Ausencia del criterio. 


\section{(3) FORMACION Y CAPACITACION DEL RRHH}

«La empresa enfatiza la utilización de procedimientos de formación y capacitación dirigidos a los colaboradores que actúan en el área de gestión internacional».

Máximo énfasis

Mínimo énfasis

\begin{tabular}{|l|l|l|l|l|}
\hline & & & & \\
\hline
\end{tabular}

En relación con el tema de la referencia ¿Qué tipo de procedimientos concretos y herramientas específicas utiliza la empresa?

Criterios orientadores para ayudar a fijar el nivel de énfasis del factor

\section{NIVEL A:}

Las personas que actúan en el área de gestión internacional poseen experiencia y sólida formación en materias de exportaciones y comercio exterior. Estos son permanentemente capacitados por la empresa y motivados adecuadamente para estimular su formación e iniciativa respecto del negocio exportador.

NIVEL B:

Las personas que actúan en el área de gestión internacional están bien calificadas y cuentan con experiencia sin embargo no reciben un nivel de capacitación ni formación constante por parte de la empresa en cuanto a las materias relativas al negocio exportador.

\section{NIVEL C:}

Las personas que actúan en el área de gestión internacional provienen de áreas en las cuales no recibieron formación adecuada en materia de exportaciones y comercio exterior, sin embargo han ganado experiencia en este campo haciendo «camino al andar», contando eso si con un nivel de apoyo en materias de capacitación más bien esporádico y discreto por parte de la empresa.

\section{NIVEL D:}

Las personas que actúan en el área de gestión internacional no tienen experiencia en lo procesos pertinentes a dicha área, dado que esta es una actividad muy incipiente en la empresa. A la fecha no se ha formado un grupo interno, en condiciones de abordar la gestión internacional de la empresa y no existen niveles formales de capacitación al respecto.

NIVEL E:

Ausencia del criterio. 


\section{(4) PLANIFICACION DE LA GESTION INTERNACIONAL}

«La empresa enfatiza la utilización de procedimientos y metodologías para el desarrollo de la planificación de su gestión internacional».

Máximo énfasis

Mínimo énfasis

\begin{tabular}{|l|l|l|l|l|}
\hline & & & & \\
\hline
\end{tabular}

En relación con el tema de la referencia ¿Qué tipo de procedimientos concretos y herramientas específicas utiliza la empresa?

Criterios orientadores para ayudar a fijar el nivel de énfasis del factor

\section{NIVEL A:}

La empresa cuenta con una metodología apropiada para el proceso de planificación exportador, abarcando los procedimientos administrativos, de comercialización y productivos. Existen normas establecidas y procedimientos claros acerca de la forma de actuar de la organización en materia internacional.

\section{NIVEL B:}

La empresa ha considerado algunos aspectos en las áreas metodológicas del plan exportador que cubren fundamentalmente los procesos del tipo administrativo y productivo.

\section{NIVEL C:}

Existen al interior de la empresa solamente métodos que cubren los aspectos administrativos del plan exportador. La metodología necesaria para cubrir el resto de los aspectos no ha sido desarrollada

\section{NIVEL D:}

Los procedimientos de planificación exportador no están incorporados en la gestión de la empresa, debiéndose recurrir a información de distintas fuentes en las ocasiones que se necesitan. No hay registros ni pautas definidas sobre aspectos claves del proceso exportador.

NIVEL E:

Ausencia del criterio. 


\section{(5) MECANISMOS DE SELECCIÓN Y EVALUACION DE MERCADOS EX- TRANJEROS}

«La empresa enfatiza la utilización de procedimientos y mecanismos formales para llevar a cabo la evaluación y selección de los mercados foráneos».

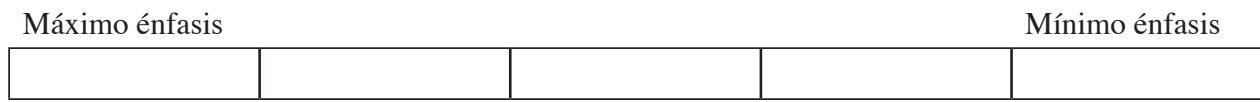

En relación con el tema de la referencia ¿Qué tipo de procedimientos concretos y herramientas específicas utiliza la empresa?

Criterios orientadores para ayudar a fijar el nivel de énfasis del factor

\section{NIVEL A:}

La empresa utiliza en forma periódica una metodología técnica y formal para llevar a cabo el proceso de selección de mercados foráneos, por cuanto dispone de sistemas de información de marketing e investigación de mercados, así como de modelos formales de evaluación de mercados extranjeros.

\section{NIVEL B:}

La empresa dispone de modelos técnicos y formales para el proceso de selección de mercados foráneos, sin embargo se superpone el poder experto y el olfato empresarial de sus directivos por sobre cualquier consideración metodológica formal al momento de evaluar y decidir.

\section{NIVEL C:}

Si bien la empresa reconoce la necesidad de contar con mecanismos formales de selección de mercados extranjeros, en la práctica no dispone ni cuenta con ellos, por lo tanto al momento de tomar decisiones al respecto, la empresa utiliza exclusivamente el poder experto y olfato empresarial.

\section{NIVEL D:}

Los procedimientos de selección de mercados extranjeros no están incorporados ni formal ni informalmente en la gestión de la empresa, debiéndose recurrir a información de distintas fuentes en las ocasiones que esta se necesita.

NIVEL E:

Ausencia del criterio. 


\section{(6) FLEXIBILIDAD Y ADAPTACIÓN DE LA ESTRATEGIA DE MARKETING INTERNACIONAL}

«La empresa enfatiza la utilización de procedimientos que le permitan flexibilizar y adaptar su estrategia de marketing internacional si el mercado o el cliente así lo requieren».

Máximo énfasis

Mínimo énfasis

\begin{tabular}{|l|l|l|l|l|}
\hline & & & & \\
\hline
\end{tabular}

En relación con el tema de la referencia ¿Qué tipo de procedimientos concretos y herramientas específicas utiliza la empresa?

Criterios orientadores para ayudar a fijar el nivel de énfasis del factor

\section{NIVEL A:}

La empresa privilegia como parte de su estrategia de marketing internacional, el uso de procedimientos que le permiten flexibilizar hasta en un $100 \%$ su mix de marketing (si el mercado o el cliente así lo requieren). Para ello la empresa dispone de la completa posibilidad de efectuar adaptaciones a sus productos, efectuar diferenciación de precios, flexibilizar su estrategia de distribución de acuerdo al mercado de destino y adaptar completamente su plan comunicacional si fuera necesario.

\section{NIVEL B:}

La empresa privilegia como parte de su estrategia de marketing internacional, el uso de procedimientos que le permiten flexibilizar hasta en un 50\% su mix de marketing (si el mercado o el cliente así lo requieren). Para ello la empresa dispone de la posibilidad parcial de efectuar adaptaciones a sus productos, efectuar un discreto nivel de diferenciación en los precios, flexibilizar parcialmente su estrategia de distribución de acuerdo al mercado de destino y adaptar parcialmente su plan comunicacional solo si fuera estrictamente necesario.

\section{NIVEL C:}

La empresa utiliza como parte de su estrategia de marketing internacional una estrategia indiferenciada (o de estandarización) para su mix de marketing.

NIVEL D:

La empresa no tiene una política formal para el desarrollo de su mix de marketing como parte de su estrategia de marketing internacional.

NIVEL E:

Ausencia del criterio. 


\section{(7) PRESENCIA DEL DEPARTAMENTO DE EXPORTACIONES (Selección múltiple)}

\section{NIVEL 1:}

La empresa cuenta con una división/departamento especializado en comercio exterior, con claras funciones y competencias en el tema de gestión internacional. Su dependencia es de la dirección superior, contando con apoyo e infraestructura así como con un personal responsable que conoce respecto de los temas relacionados con exportaciones e internacionalización del negocio vitivinícola.

\section{NIVEL 2:}

La empresa cuenta con personal dependiente directamente de la dirección superior, con conocimientos de los mercados objetivos, no obstante se carece de la infraestructura departamental necesaria.

\section{NIVEL 3:}

La empresa exporta sin embargo las funciones correspondientes han sido desempeñadas por el departamento de ventas interno de la misma u otras áreas de la empresa.

\section{NIVEL 4:}

No existe la función de exportaciones como parte de la organización de la empresa. El ejecutivo superior maneja esta área incipiente, contando con eventual soporte de algunos jefes de área que se estima conocen algo del tema.

\section{NIVEL 5:}

Ninguna de las anteriores. 


\section{(8) GESTION DE REDES}

(Selección múltiple)

\section{NIVEL 1:}

La empresa ha generado bases de operación en el exterior, localizadas en distintos mercados objetivo, por medio de tejer redes empresariales que le han permitido conectividad y cooperación con empresas asociadas, proveedoras y clientes. Con ello ha logrado mejorar su posición exportadora en forma considerable.

\section{NIVEL 2:}

La empresa comprende el beneficio asociado al fenómeno de la gestión de redes empresariales para facilitar el proceso de internacionalización, sin embargo su planteamiento práctico ha sido muy incipiente y escaso. En términos prácticos no ha logrado desarrollar una red eficiente de trabajo por lo que ha tenido que optar preferentemente por el desarrollo de bases de operación en mercados extranjeros con personal propio y con retroalimentación sistemática a la casa matriz de la empresa.

\section{NIVEL 3:}

La empresa no visualiza en su totalidad el beneficio asociado al fenómeno de gestión de redes empresariales como mecanismo para facilitar el proceso de internacionalización, por lo tanto no ha realizado esfuerzos concretos por beneficiarse del sistema.

\section{NIVEL 4:}

La empresa no se interesa en gestionar por medio del mecanismo de gestión de redes empresariales si no por el contrario privilegia el desarrollo de experiencias internacionales del tipo «autodidactas y en solitario».

\section{NIVEL 5:}

Ninguna de las anteriores. 


\section{(9) EXPERIENCIA INTERNACIONAL}

(Selección múltiple)

\section{NIVEL 1:}

La empresa ha formulado la totalidad de su estrategia exportadora basándose en la abundante experiencia previa que detenta como organización así como su equipo directivo. Lo anterior le ha permitido beneficiarse de la disminución de costos asociados al proceso de aprendizaje del negocio internacional así como de poder mejorar considerablemente su toma de decisiones en la selección y penetración de mercados extranjeros.

\section{NIVEL 2:}

Si bien la empresa exporta, esta detenta un nivel de experiencia internacional discreta, aspecto que le ha dificultado poder definir un plan de gestión internacional eficiente y bien calibrado, afectando el nivel de competitividad requerido por la oferta exportable así como la correcta selección y penetración de mercados extranjeros.

\section{NIVEL 3:}

La empresa manifiesta voluntad exportadora pero carece de experiencia considerable en este campo. Tiene una idea general de los mercados en los cuales sus productos podrían competir pero sin definirlos. No ha asistido a ferias, ni se ha detenido a estudiar con profundidad los productos con los cuales competir ni los precios a los que se deberían vender para tener éxito.

\section{NIVEL 4:}

Existe interés por explorar posibilidades de exportar en forma activa, estimándose que se estaría en condiciones de hacerlo, sin embargo no se cuenta ni con la experiencia ni el conocimiento suficiente como para poder hacerlo. La empresa exporta en forma esporádica.

\section{NIVEL 5:}

Ninguna de las anteriores. 


\section{(10) ADAPTACION DE LA OFERTA EXPORTABLE}

(Selección múltiple)

\section{NIVEL 1:}

La empresa privilegia como parte de su estrategia de marketing internacional, procedimientos que le permiten efectuar una total adaptación de su oferta exportable (si el mercado o el cliente así lo requieren). Para ello la empresa dispone de variadas líneas de productos en cuanto a amplitud y niveles de profundidad así como con un servicio de monitoreo periódico de la satisfacción de sus cliente aspectos que le permiten corregir desviaciones tanto del producto final como de los procedimientos involucrados.

\section{NIVEL 2:}

La empresa privilegia como parte de su estrategia de marketing internacional, procedimientos que le permiten efectuar una parcial adaptación de su oferta exportable (si el mercado o el cliente así lo requieren). El diseño y la calidad de los productos es satisfactorio sin embargo no detenta una capacidad de respuesta inmediata para corregir desviaciones de la oferta exportable.

\section{NIVEL 3:}

La empresa privilegia como parte de su estrategia de marketing internacional una estrategia indiferenciada (o de estandarización) para su oferta exportable.

\section{NIVEL 4:}

La empresa no tiene una política formal para el desarrollo de su oferta exportable como parte de su estrategia de marketing internacional.

\section{NIVEL 5:}

Ninguna de las anteriores. 


\section{(11) DIFERENCIACION DE LAS POLITICAS DE PRECIO INTERNACIONAL (Selección múltiple)}

\section{NIVEL 1:}

La empresa privilegia como parte de su estrategia de marketing internacional, una política de diferenciación total de precios de acuerdo a las características particulares de los diferentes mercados y de acuerdo a la disposición a pagar de los distintos segmentos de clientes.

\section{NIVEL 2:}

La empresa privilegia como parte de su estrategia de marketing internacional, una política de diferenciación parcial de precios (solo a veces diferencia sus precios) de acuerdo a las características particulares de los diferentes mercados y a la disposición a pagar de los distintos segmentos de clientes.

\section{NIVEL 3:}

La empresa privilegia como parte de su estrategia de marketing internacional una estrategia indiferenciada o de estandarización de precios. A todos los clientes y mercados les vende a igual nivel de precio.

\section{NIVEL 4:}

La empresa no tiene una política de precios establecida para su estrategia de marketing internacional.

\section{NIVEL 5:}

Ninguna de las anteriores. 


\section{(12) FLEXIBILIDAD DEL PLAN DE COMUNICACION INTERNACIONAL} (Selección múltiple)

\section{NIVEL 1:}

La empresa privilegia como parte de su estrategia de marketing internacional, procedimientos que le permiten una total adaptación de su plan comunicacional si el mercado así lo requiere. Para ello cuenta con apoyo de agencias e intermediarios en los mercados de destino que le permiten calibrar los contenidos de su mensaje comunicacional así como los medios de difusión del mensaje.

\section{NIVEL 2:}

La empresa privilegia como parte de su estrategia de marketing internacional, procedimientos que le permiten una parcial adaptación de su plan comunicacional si el mercado así lo requiere. Para ello cuenta con apoyo de agencias e intermediarios en los mercados de destino que le permiten calibrar los contenidos de su mensaje comunicacional manteniendo los criterios dictados por la casa matriz.

\section{NIVEL 3:}

La empresa privilegia como parte de su estrategia de marketing internacional una estrategia indiferenciada o de estandarización en su plan comunicacional (todos por igual).

\section{NIVEL 4:}

La empresa no tiene una política comunicacional establecida dentro de su estrategia de marketing internacional.

\section{NIVEL 5:}

Ninguna de las anteriores. 


\section{(13) DESARROLLO DE CANALES COLABORATIVOS DE DISTRIBUCIÖN INTERNACIONAL}

(Selección múltiple)

\section{NIVEL 1:}

La empresa ha desarrollado canales robustos de intermediación en los distintos mercados donde comercializa sus productos.

\section{NIVEL 2:}

La empresa ha logrado desarrollar canales incipientes de intermediarios en los distintos mercados donde comercializa sus productos.

\section{NIVEL 3:}

La empresa no está interesada en privilegiar canales de intermediación, por el contrario prefiere favorecer la comercialización de sus productos por medio de grandes compradores que manejan redes propias de intermediación.

\section{NIVEL 4:}

La empresa no dispone de una política de intermediación y desarrollo de canales de distribución como parte de su estrategia de marketing internacional.

\section{NIVEL 5:}

Ninguna de las anteriores. 


\section{(14) GESTIÓN ESTRATÉGICA EMPRESARIAL}

(Selección múltiple)

\section{NIVEL 1:}

La empresa cuenta con una definición clara y precisa respecto de su visión y misión así como de sus objetivos estratégicos, los cuales engarzan en forma lógica y consistente con su estrategia internacional. Procura además la incorporación de capacidades distintivas y está permanentemente atenta a las variaciones del entorno comercial y tecnológico.

\section{NIVEL 2:}

La empresa detenta lineamientos básicos de orientación, los cuales se sustentan en la percepción de sus principales ejecutivos. La visión, misión y estrategia es de mediano plazo y no siempre se encuentra correctamente alineada con los fundamentos de su estrategia internacional.

\section{NIVEL 3:}

La proyección del desarrollo de la empresa es parcial y de corto plazo. Las definiciones de objetivos estratégicos son incompletas, ya que no considera la totalidad de factores de éxito para un desarrollo sostenido.

\section{NIVEL 4:}

Los principios de estrategia no están establecidos en la empresa, existiendo una visión parcial de corto plazo. La operación se conduce en términos de reacción frente a las evoluciones de la demanda, sin anticipación alguna a los cambios.

\section{NIVEL 5:}

Ninguna de las anteriores. 


\section{(15) INTEGRACIÓN DE ÁREAS FUNCIONALES AL PROCESO EXPORTADOR (Selección múltiple)}

\section{NIVEL 1:}

Las distintas áreas funcionales de la empresa ligadas al proceso exportador conocen claramente sus funciones y actúan en forma coordinada y con gran sentido de equipo para un apoyo eficaz a los objetivos trazados. La coordinación es eficiente y la colaboración espontánea.

\section{NIVEL 2:}

Si bien, las diferentes áreas funcionales conocen sus funciones dentro del proceso exportador, no existe una coordinación total ni claridad en los objetivos finales buscados por la empresa.

\section{NIVEL 3:}

Las áreas funcionales trabajan solamente debido a su sentido de equipo y de responsabilidad ante el futuro de la empresa. Suelen ocurrir excusas tales como «esto no cae bajo mi responsabilidad»o «nunca supe realmente lo que se buscaba».

\section{NIVEL 4:}

No se produce una efectiva coordinación entre las diferentes funciones de la empresa, de forma que se resiente el logro de las metas de desarrollo de las operaciones internacionales. Es necesario un fuerte impulso superior para armonizar el comportamiento y desempeño funcional.

\section{NIVEL 5:}

Ninguna de las anteriores. 


\section{(16) ABASTECIMIENTO DE MATERIAS PRIMAS}

(Selección múltiple)

\section{NIVEL 1:}

Los insumos de materiales están disponibles para la empresa, existiendo oferta en el mercado en la calidad requerida y a precios competitivos respecto de los prevalecientes en el exterior. La empresa cuenta con una adecuada cantidad de proveedores de insumos básicos, con los cuales mantiene excelentes relaciones comerciales. Con éstos se pactan acuerdos de suministro por períodos determinados, los cuales tienen grados de flexibilidad adecuados.

\section{NIVEL 2:}

Existe suficiente oferta en el mercado interno, sin embargo hay muy pocos proveedores de insumos, en la calidad y precios requeridos. Lo anterior presiona a la empresa en cuanto a tener que estrechar la colaboración con estos proveedores con el fin de no tener que ceder poder de negociación innecesariamente.

\section{NIVEL 3:}

Existen dificultades para la obtención oportuna de los insumos por lo que una orden de exportación importante requeriría de plazos prolongados para su correcto cumplimiento.

\section{NIVEL 4:}

El abastecimiento de materias primas presenta algunas dificultades para la empresa, derivadas de la calidad requerida y la oferta concentrada en muy pocos proveedores. Los precios que se pueden obtener no son competitivos frente a los fabricantes en el exterior. La empresa no utiliza convenios de abastecimiento.

\section{NIVEL 5:}

Ninguna de las anteriores. 


\section{(17) SOPORTE INSTITUCIONAL EN EL EXTERIOR \\ (Selección múltiple)}

\section{NIVEL 1:}

La empresa recibe un adecuado, eficiente y eficaz nivel de apoyo proveniente de instituciones de fomento exportador y de promoción al comercio bilateral.

\section{NIVEL 2:}

La empresa recibe buena información pero en forma esporádica proveniente de instituciones de fomento exportador y de promoción al comercio bilateral.

\section{NIVEL 3:}

La empresa recibe información parcial e incompleta proveniente de instituciones de fomento exportador y de promoción al comercio bilateral

\section{NIVEL 4:}

La empresa no dispone de una vinculación efectiva con entidades de promoción y/o fomento de exportaciones. El apoyo recibido es indirecto y de un nivel de eficiencia primario. No se cuenta con estadísticas confiables ni con orientaciones estratégicas de mercado.

\section{NIVEL 5:}

Ninguna de las anteriores. 


\section{(18) GESTIÓN MEDIOAMBIENTAL}

(Selección múltiple)

\section{NIVEL 1:}

La empresa concede gran importancia al equilibrio con el medio ambiente para eliminar las causas de la contaminación. Existe un adecuado tratamiento de los desechos, los que han sido minimizados en su origen. Se cuenta con certificaciones de norma internacional del tipo ISO 14.000 o al menos se encuentra en etapas de preparación para su obtención.

\section{NIVEL 2:}

La empresa concede una importancia discreta al tema ambiental sin embargo ha preparado planes para hacer frente a este problema y ha comenzado a implantar sistemas de mejora ambiental.

NIVEL 3:

La empresa está consciente de los problemas ambientales que genera, ha evaluado sus alternativas y los costos envueltos sin tomar aún decisiones.

NIVEL 4:

La empresa no ha evaluado sus problemas, sus alternativas, los costos envueltos y no tiene planes correctivos. Actúa por reacción cuando los problemas se presentan.

NIVEL 5:

Ninguna de las anteriores. 


\section{(19) INTEGRACIÓN A LOS OBJETIVOS ESTRATÉGICOS INTERNACIONALES (Selección múltiple)}

\section{NIVEL 1:}

Existe pleno conocimiento de la estrategia internacional al interior de la empresa, con una adhesión total a los fines perseguidos por parte del personal de distintas áreas, en la dirección de apoyo para el logro de las metas establecidas

\section{NIVEL 2:}

La empresa, a pesar de tener una estrategia internacional definida, no ha sabido comunicarla y difundirla a todos los niveles de la organización y tampoco implementarla en términos operativos y funcionales.

\section{NIVEL 3:}

La definición de los objetivos estratégicos de índole internacional es más bien genérica e imprecisa generando confusiones en el personal.

\section{NIVEL 4:}

Existe un desconocimiento general de los objetivos estratégicos de la empresa, de manera que la colaboración del personal para el logro de las metas es prácticamente nula.

\section{NIVEL 5:}

Ninguna de las anteriores. 


\section{Gipuzkoaren kanpo harremanak, esku onetan.}

\section{Las relaciones externas de Gipuzkoa, en buenas manos.}

\section{Les relations externes de Gipuzkoa, en bonnes mains.}

Gipuzkoako Foru Aldundia

Diputación Foral de Gipuzkoa 